Article

\title{
Biooxidation of Ciguatoxins Leads to Species-Specific Toxin Profiles
}

\author{
Tsuyoshi Ikehara ${ }^{1, *}$, Kyoko Kuniyoshi ${ }^{2}$, Naomasa Oshiro ${ }^{2}$ and Takeshi Yasumoto ${ }^{3}$ \\ 1 Department of Food Science and Technology, National Fisheries University, 2-7-1 Nagata-honmachi, \\ Shimonoseki, Yamaguchi 759-6595, Japan \\ 2 National Institute of Health Sciences, 1-18-1 Kamiyoga, Setagaya, Tokyo 158-8501, Japan; \\ k-kuniyoshi@nihs.go.jp (K.K.); n-oshiro@nihs.go.jp (N.O.) \\ 3 Japan Food Research Laboratories, 6-11-10 Nagayama, Tama, Tokyo 206-0025, Japan; yasumotot@jfrl.or.jp \\ * Correspondence: ikehara@fish-u.ac.jp; Tel.: +81-83-227-3916
}

Academic Editor: Irina Vetter

Received: 2 June 2017; Accepted: 27 June 2017; Published: 29 June 2017

\begin{abstract}
Ciguatoxins (CTXs) contaminate fish worldwide and cause the foodborne illness ciguatera. In the Pacific, these toxins are produced by the dinoflagellate Gambierdiscus toxicus, which accumulates in fish through the food chain and undergoes oxidative modification, giving rise to numerous analogs. In this study, we examined the oxidation of CTXs in vitro with liquid chromatography-tandem mass spectrometry (LC-MS/MS) analysis using reference toxins, and found that CTX4A, CTX4B, and CTX3C, which are produced by the alga, are oxidized to the analogs found in fish, namely CTX1B, 52-epi-54-deoxyCTX1B, 54-deoxyCTX1B, 2-hydroxyCTX3C, and 2,3-dihydroxyCTX3C. This oxidation was catalyzed by human CYP3A4, fish liver S9 fractions, and microsomal fractions prepared from representative ciguateric fishes (Lutjanus bohar, L. monostigumus, and Oplegnathus punctatus). In addition, fish liver $S 9$ fractions prepared from non-ciguateric fishes (L. gibbus and L. fulviflamma) in Okinawa also converted CTX4A and CTX4B to CTX1B, 54-deoxyCTX1B, and 52-epi-54-deoxyCTX1B in vitro. This is the first study to demonstrate the enzymatic oxidation of these toxins, and provides insight into the mechanism underlying the development of species-specific toxin profiles and the fate of these toxins in humans and fish.
\end{abstract}

Keywords: ciguatera; ciguatoxins; in vitro oxidation; fish liver S9; Cyp3A4

\section{Introduction}

Ciguatera fish poisoning (CFP) is a clinical syndrome that causes neurological symptoms in patients and affects more than 50,000 people per year [1-5]. This foodborne illness results from eating fish from warm water regions around the world that contain natural marine toxins that are collectively known as ciguatoxins (CTXs). CTXs are produced by the benthic dinoflagellate initially assigned Gambierdiscus toxicus with reassignment later to G. polynesiensis, enter the food chain via herbivorous fish and subsequently accumulate in the tissue of hundreds of species of fish [6-8].

Chemical studies performed on the dinoflagellate and fish from the Pacific have identified up to 23 analogs of CTXs, all of which contain contiguous ether rings aligned in a ladder shape $[9,10]$. However, the backbone skeletons of these toxins differ, allowing them to be classified into two distinctive types: CTX1B and CTX3C (Figure 1). It has been shown that the oxygenated congeners CTX1B and 54-deoxyCTX1B are more toxic than CTX4A and 4B in mice [7,11-14]. Furthermore, a distinction has been noted between the toxins that are produced by G. toxicus and those that are found in fish, strongly suggesting that the original toxins are metabolically-oxidized in fish, mostly at the terminal parts of the molecule; and a recent study showed that various fish from Okinawa and some other regions retained species-specific toxin profiles over a 20 -year time period [15,16], implying 
that a genetic factor may be involved in shaping their toxin profiles. However, this hypothetical biooxidation of CTXs has remained untested due to the unavailability of pure toxins for use as substrates and for identifying the products.

Cytochrome p450 (CYP) enzymes constitute a superfamily of many different monooxygenases that play a pivotal role in drug metabolism [17]. These enzymes are present in all living organisms and are expressed in many tissues in vivo, but are most abundant in the mammalian liver. Thus, the liver S9 and microsomal fractions include drug metabolizing enzymes such as CYP enzymes, and are mainly used to test for the metabolism of alien substances, drug interactions, and the formation of covalent bonds between drugs [18-20].

In this study, we aimed to verify the bio-oxidation process of CTXs in vitro using CTX4A/4B and CTX3C as substrates, and recombinant human CYP3A4 (rhCYP3A4), fish liver S9 fractions, and microsomal fractions as oxidizing enzymes. The reaction products were identified by liquid chromatography-tandem mass spectrometry (LC-MS/MS) analysis using reference toxins, which unambiguously confirmed the production of CTX1B, 54-deoxyCTX4B, 52-epi-54-deoxyCTX1B, 2-hydroxyСТХ3C, and 2,3-dihydroxyCТХ3С. Our findings shed light on the mechanism that leads to the characteristic toxin profiles in fish species and also enrich our knowledge of the dynamic fate of these toxins.

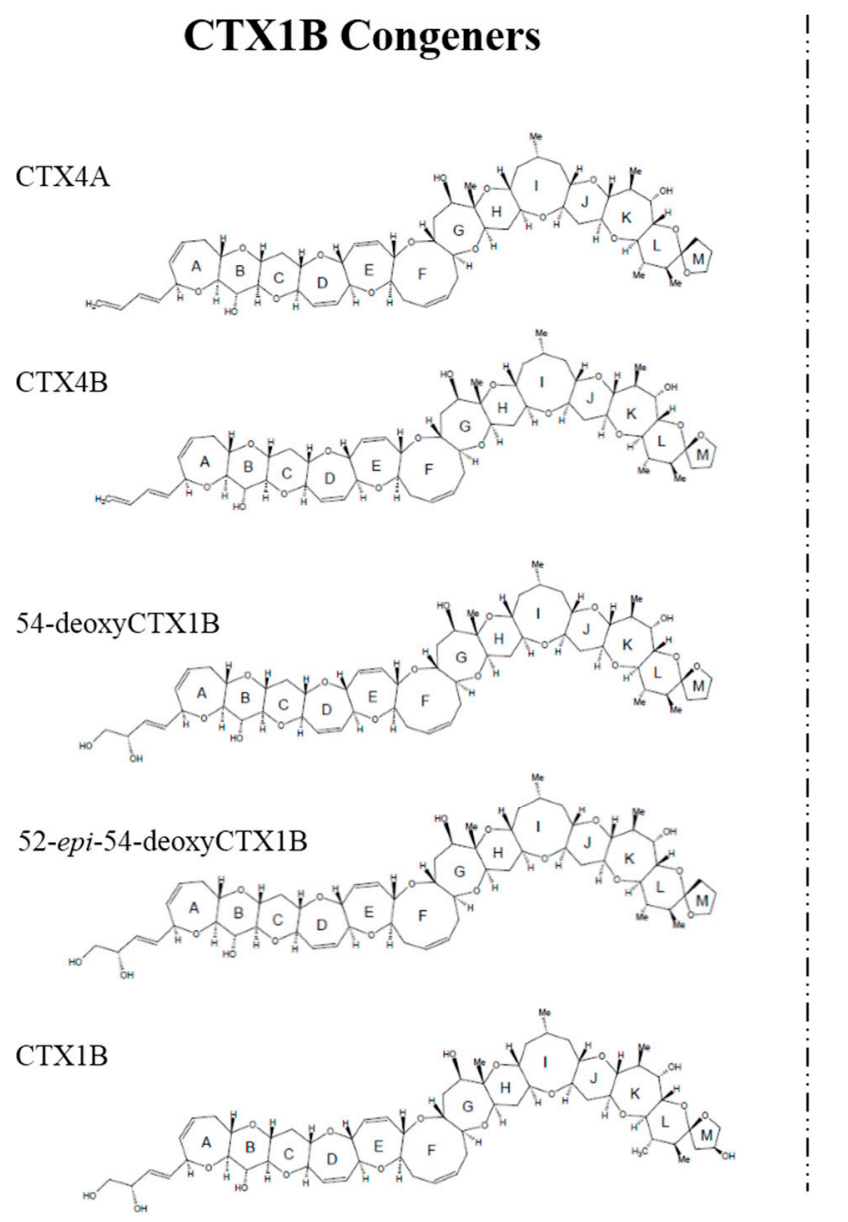

\section{CTX3C Congeners}

CTX3C
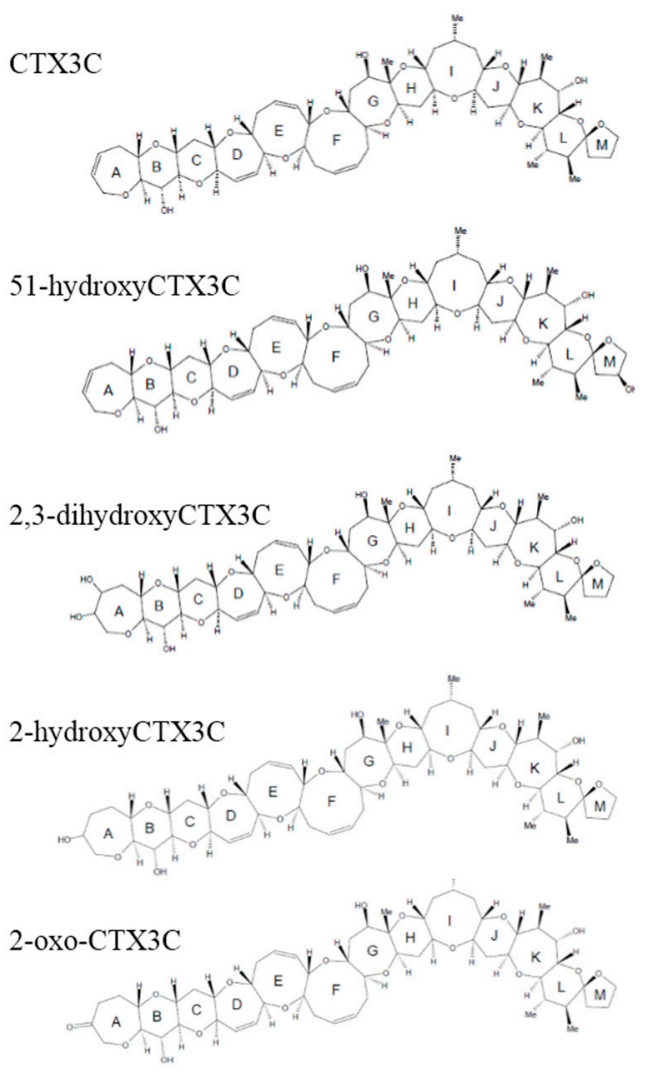

Figure 1. Structures of various ciguatoxins (CTXs). 


\section{Results}

\subsection{In Vitro Oxidation of CTXs by rhCYP3A4}

To verify the biooxidation of CTXs, we attempted to oxidize the substrates CTX4A, CTX4B, and CTX3C in vitro (Figure S1a,b), and identified the reaction products by LC-MS/MS analysis using reference toxins (Figure S1c). The abbreviations and the $[\mathrm{M}+\mathrm{Na}]^{+}(m / z)$ value of each CTX congener is shown in Table 1. To establish the experimental conditions, we first attempted in vitro oxidation using rhCYP3A4 and human liver microsomes because CYP3A4 is one of many human CYP enzymes that plays a central role in drug metabolism, and biotransforms a wide range of endogenous and exogenous compounds including drugs, toxins, and pollutants. We found that incubation of CTX3C with rhCYP3A4 produced 51-hydroxyCTX3C (Figure 2); incubation of CTX4A/4B with rhCYP3A4 produced CTX1B, M-seco-CTX4A/4B, and 7-hydroxyCTX4A/4B (Figure S2); and incubation of CTX4A/4B with human liver microsomes including CYP3A4 produced CTX1B, 52-epi-54-deoxyCTX1B, 54-deoxyCTX1B, and M-seco-CTX4A/4B, none of which were detected when microsomes that had been inactivated by heat-treatment were used (Figure S3). These results indicate that rhCYP3A4 and human liver microsomes, including CYP3A4, can be successfully used for in vitro CTX oxidation.
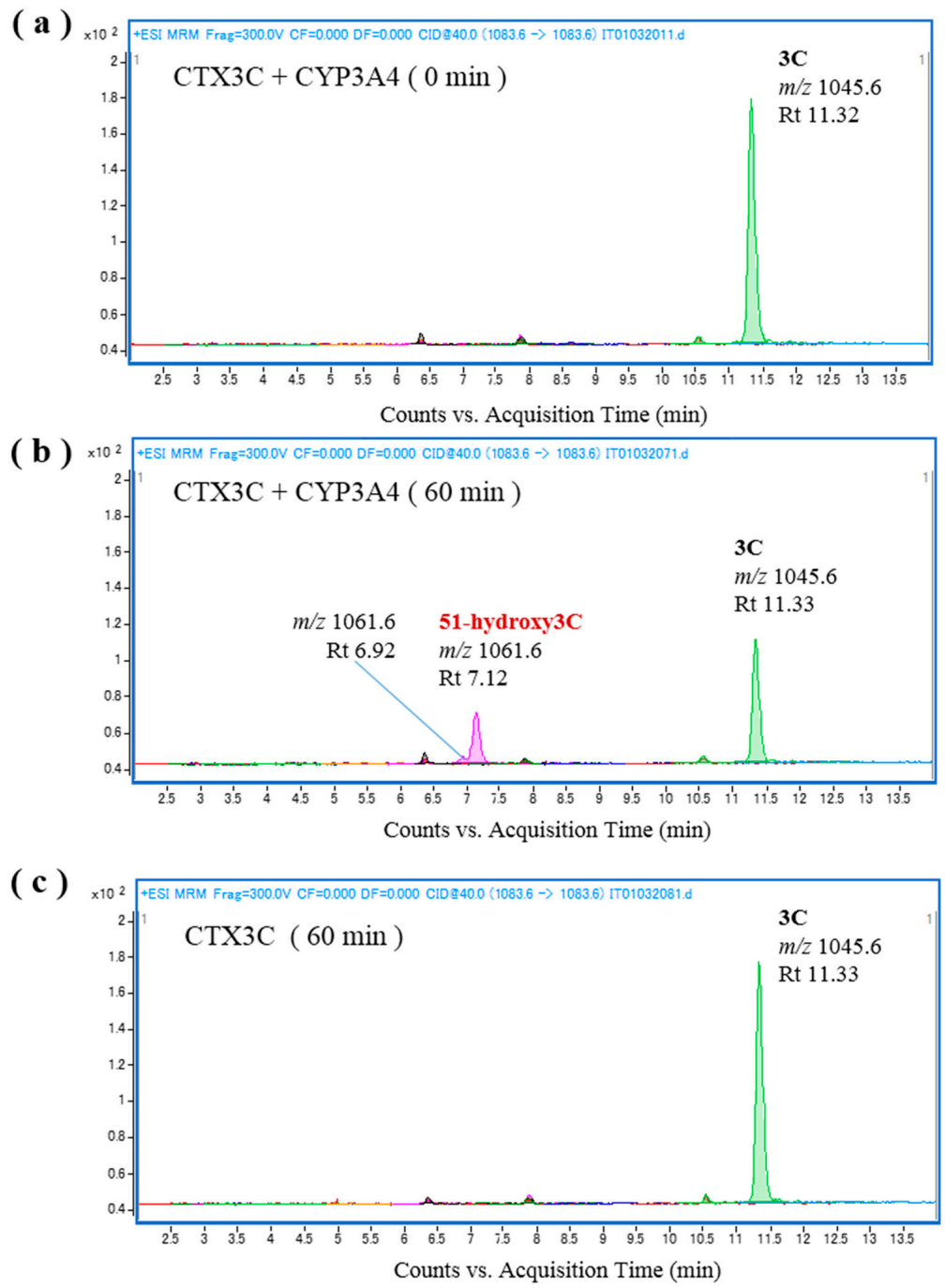

Figure 2. Chromatograms of the in vitro oxidation products of ciguatoxin-3C (CTX3C) following incubation with recombinant human CYP3A4 (rhCYP3A4) at $37^{\circ} \mathrm{C}$. Products are shown (a) at $0 \mathrm{~min}$, (b) at $60 \mathrm{~min}$, and (c) at $60 \mathrm{~min}$ without rhCYP3A4. 
Table 1. The abbreviations and the $[\mathrm{M}+\mathrm{Na}]^{+}(m / z)$ value of each CTX congener.

\begin{tabular}{|c|c|c|c|c|c|}
\hline Abbreviations & CTX Congeners & {$[\mathrm{M}+\mathrm{Na}]^{+} \mathrm{m} / z$} & Abbreviations & CTX Congeners & {$[\mathrm{M}+\mathrm{Na}]^{+} \mathrm{m} / z$} \\
\hline $4 \mathrm{~A}$ & СТХ4A & 1083.6 & $3 C$ & СТХЗС & 1045.6 \\
\hline $4 \mathrm{~B}$ & СТХ4В & 1083.6 & 2-охо3C & 2-охоСТХЗС & 1061.6 \\
\hline $\mathrm{M}-\sec 04 \mathrm{~A} / 4 \mathrm{~B}$ & M-secoCTХ4A/CTХ4B & 1101.6 & 2-охо-ері-3С & 2-охо-ері-СТХЗС & 1061.6 \\
\hline deoxy1B & 54-deoxyCTX1B & 1117.6 & 2,3-dihydroxy3C & 2,3-dihydroxyCTХЗC & 1079.6 \\
\hline epi-deoxy1B & 52-ерi-54-deoхуСТХ1B & 1117.6 & 51-hydroxy3C & 51-hydroxyCТХЗС & 1061.6 \\
\hline 1B & СТХ1B & 1133.6 & 2-hydroxy3C & 2-hydroxyCТХЗС & 1063.6 \\
\hline 4-hydroxy-7-oxo-1B & 4-hydroxy-7-oxo-СТХ1В & 1167.6 & & & \\
\hline
\end{tabular}

\subsection{In Vitro Oxidation of CTXs by Fish Liver S9 from Cigauteric and Non-Ciguateric Fishes}

The CTX oxidation activity of fish liver extracts was examined using the experimental conditions that were established in the in vitro oxidation experiment (see above). We prepared fish liver S9 fractions from 18 fish specimens belonging to three representative ciguateric fish species (L. bohar, L. monostigma, and O. punctatus) and two non-ciguateric fish species (L. gibbus and L. fulviflamma) (Table S1). These fish were collected from the Ryukyu Islands in Okinawa Prefecture, where large carnivores are frequently toxic, but there have been no observed cases of CFP in L. fulviflamma and L. gibbus [21].

The fish liver S9 fraction was initially prepared from $L$. bohar liver homogenate because bio-oxidation activity in this fish was expected to be high due to snappers containing the oxidative analogs CTX1B, 52-epi-54-deoxyCTX1B, and 54-deoxyCTX1B, and L. bohar reportedly being the most common reef fish that is implicated in CFP poisoning in Okinawa [21]. As shown in Figure 3a, incubation of a mixture of the substrates CTX4A and CTX4B with L. bohar liver S9 as an oxidation catalyzing system at $37^{\circ} \mathrm{C}$ for $60 \mathrm{~min}$ led to the production of 52-epi-54-deoxyCTX1B and 54-deoxyCTX1B, neither of which were contained in the substrates or the fish liver S9 fraction (Figure $3 \mathrm{~b}, \mathrm{c}$ ). Oxidation of the CTXs was also detected when a further fractionated microsomal fraction was used (Figure 3d). These results indicate that 52-epi-54-deoxyCTX1B and 54-deoxyCTX1B were produced by the in vitro oxidation of CTX4A/4B by fish liver S9, suggesting that some of the CYP family enzymes that are abundant in the fish liver have oxidation activity.

( a )

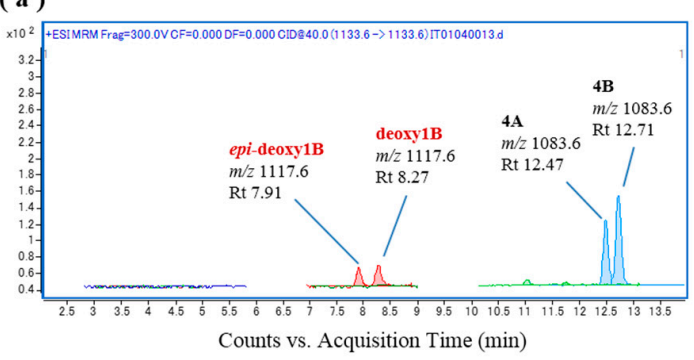

(c)

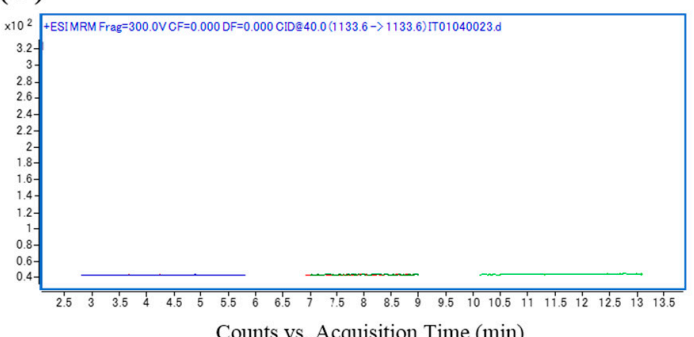

( b )

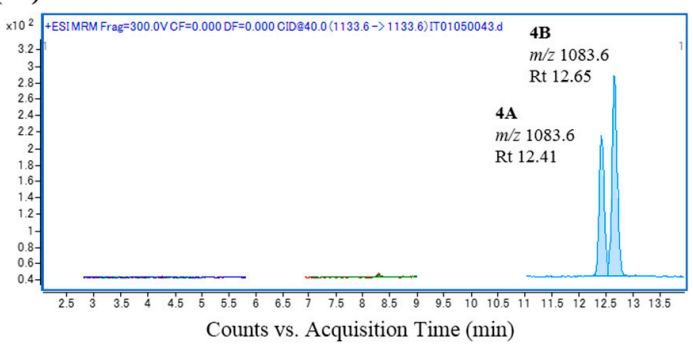

(d)

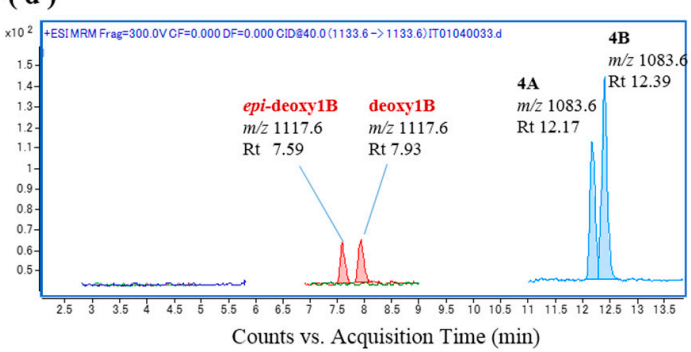

Figure 3. Chromatograms of the in vitro oxidation products of a ciguatoxin- $4 \mathrm{~A} / 4 \mathrm{~B}(\mathrm{CTX} 4 \mathrm{~A} / 4 \mathrm{~B})$ mixture following incubation with the liver S9 and microsomal fractions of L. bohar; (a) L. bohar liver S9 and CTX4A/4B, (b) CTX4A/4B, (c) L. bohar liver S9, and (d) L. bohar liver microsomal fraction and CTX4A/4B. 
To examine individual differences in CTX oxidation activity in L. bohar, liver S9 fractions were prepared from four additional specimens (see Table S1) and were used for in vitro CTX oxidation. In all samples but one, 52-epi-54-deoxyCTX1B and 54-deoxyCTX1B were again produced following incubation with the L. bohar S9 fractions (Figure S4a-d), and the fish liver S9 fractions were confirmed to be free of these toxins (Figure S5). Furthermore, when CTX3C was incubated with the L. bohar S9 fractions, 2,3-dihydroxyCTX3C was produced (Figure 4a,b) and it was confirmed that the CTX3C substrate was free of these analogs (Figure $4 c, d$ ). Tentative assignment in the absence of reference is shown with an asterisk.

( a )
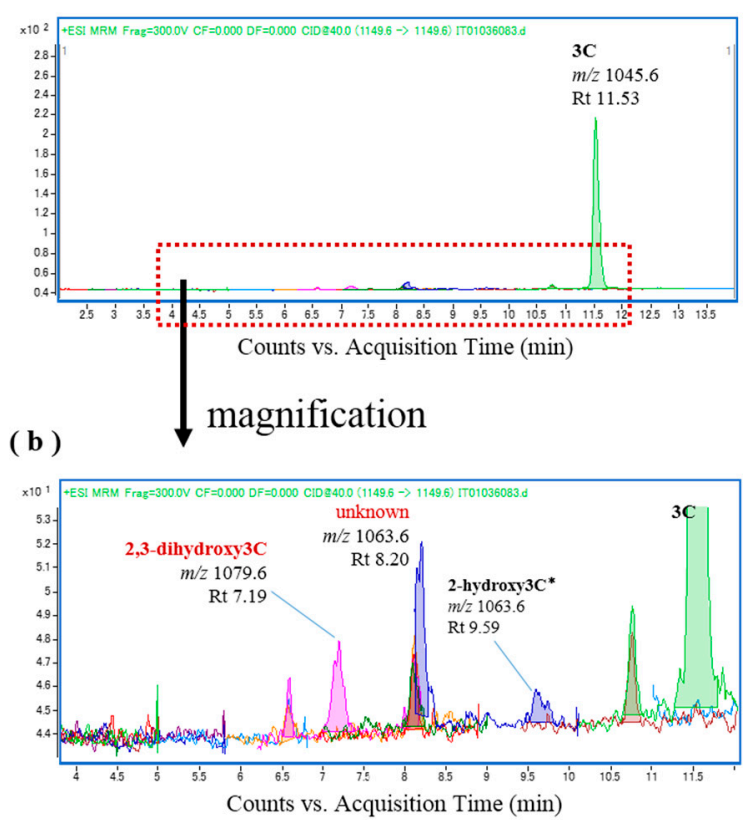

(c)
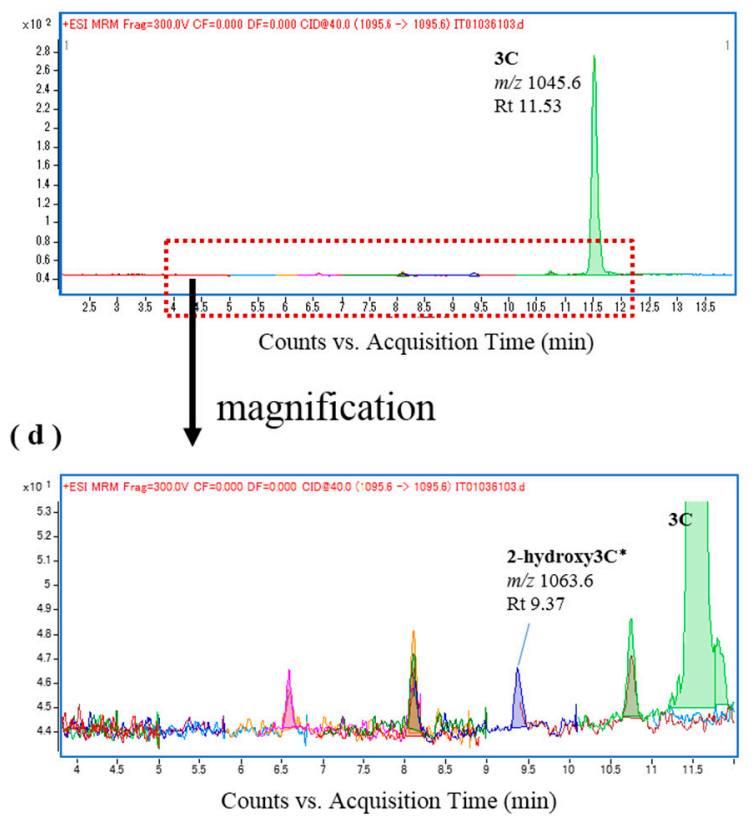

Figure 4. Chromatograms of the in vitro oxidation products of ciguatoxin-3C (CTX3C) following incubation with the liver S9 fraction of L. bohar; (a,b) L. bohar liver S9 and CTX3C; and (c,d) CTX3C.

Additional representative ciguateric fishes (L. monostigma and O. punctata) were then used to prepare liver S9 fractions for the in vitro oxidation of CTXs. We found that incubation of CTX4A/4B and CTX3C with the L. monostigma S9 fraction produced CTX1B, 52-epi-54-deoxyCTX1B, 54-deoxyCTX1B, and 2-hydroxyCTX3C (Figure 5), while incubation of these substrates with the O. punctata S9 fraction produced CTX1B, 52-epi-54-deoxyCTX1B, 54-deoxyCTX1B, M-seco-CTX4A/4B, 2,3-dihydroxyCTX3C, and 51-hydroxyCTX3C (Figure 6). Both the L. monostigma and O. punctatus S9 fractions were confirmed to be free of these toxins.

Finally, the in vitro CTX oxidation activities of the liver S9 fractions of the non-ciguateric fishes L. gibbus and L. fulviflamma were evaluated. Interestingly, although the substrates CTX4A and CTX4B were markedly reduced, only very low levels of CTX1B, 54-deoxyCTX1B, and 52-epi-54-deoxyCTX1B were produced following incubation with the liver S9 fractions of these species (Figures 7 and 8), none of which were contained in the original fractions. Comparison of the peak areas of the CTXs on the chromatograms for L. bohar, L. gibbus, and L. fulviflamma suggested that CTX4A and CTX4B were converted into some other metabolite that differed from the reference toxins (Figure 9). 
( a )

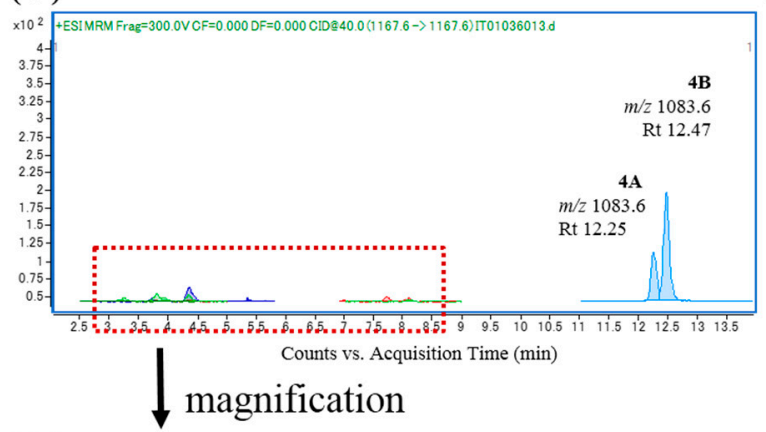

(b)

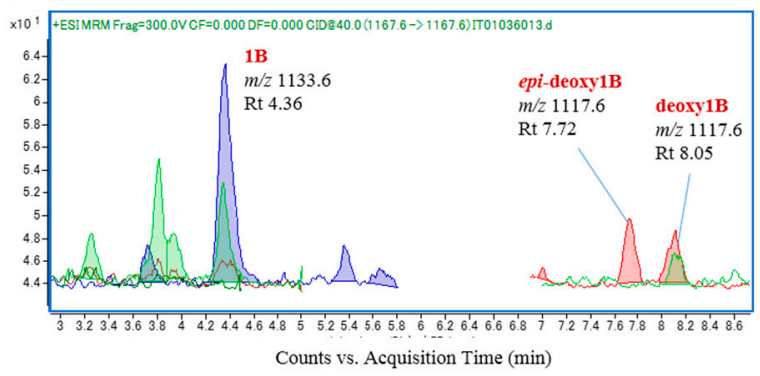

(c)

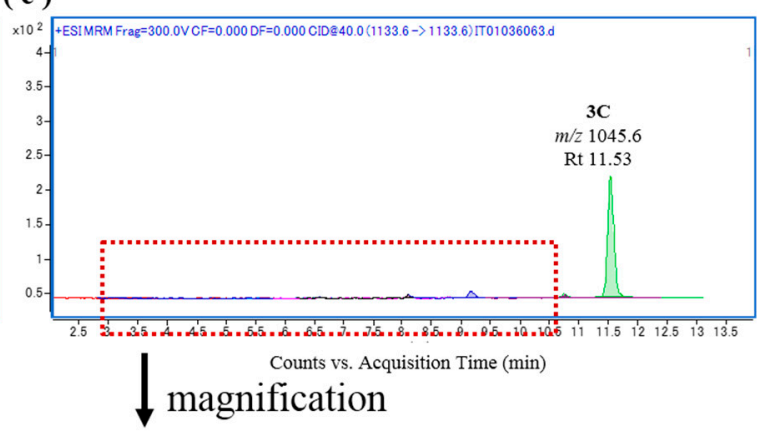

(d)

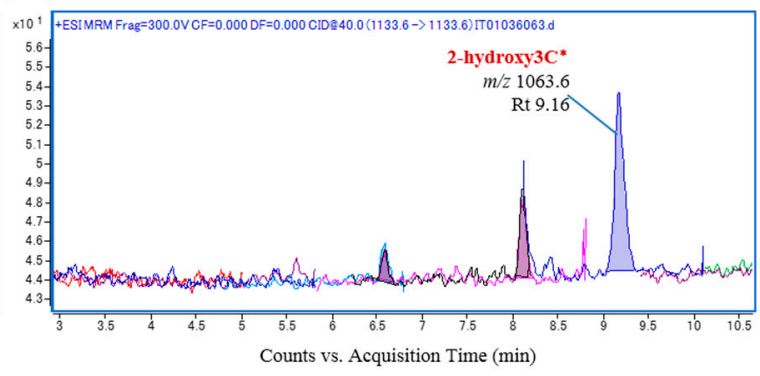

Figure 5. Chromatograms of the in vitro oxidation products of ciguatoxin- $4 \mathrm{~A} / 4 \mathrm{~B}(\mathrm{CTX} 4 \mathrm{~A} / 4 \mathrm{~B})$ and CTX3C following incubation with the liver S9 fraction of L. monostigma; $(\mathbf{a}, \mathbf{b})$ L. monostigma liver S9 and CTX4A/4B; and (c,d) L. monostigma liver S9 and CTX3C.

(a)

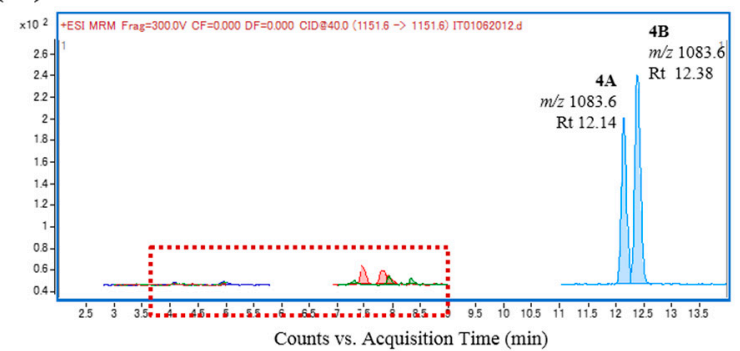
Counts vs. Acquisition Time (min)

(b )

magnification

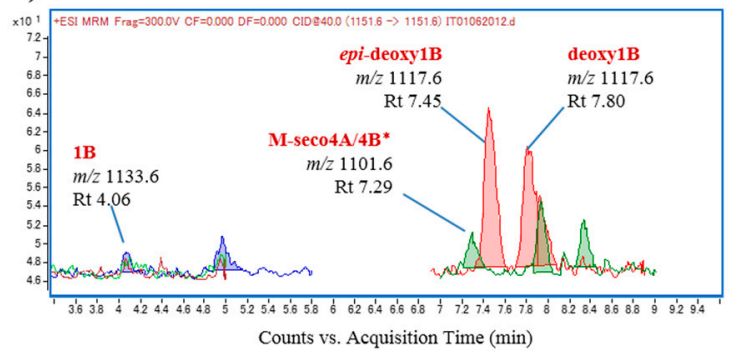

(c)

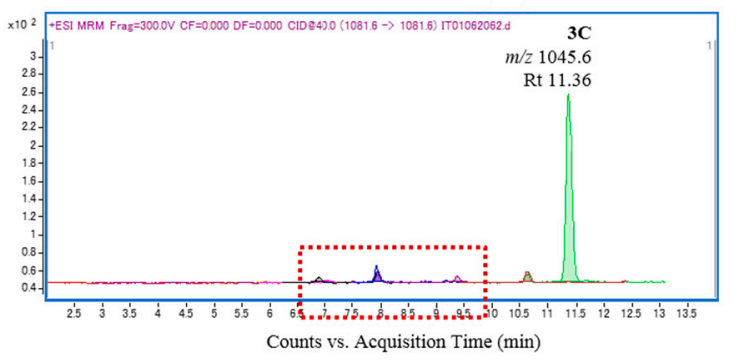

( d )

$\downarrow$ magnification

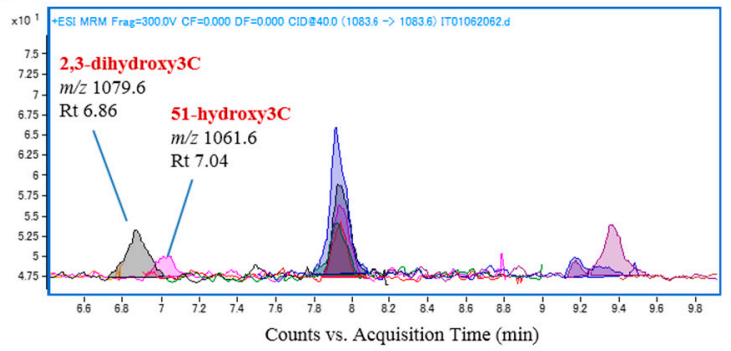

Figure 6. Chromatograms of the in vitro oxidation products of ciguatoxin-4A/4B (CTX4A/4B) and CTX3C following incubation with the liver S9 fraction of O. punctatus; $(\mathbf{a}, \mathbf{b})$ O. punctatus liver S9 and CTX4A/4B; and (c,d) O. punctatus liver S9 and CTX3C. 
(a)

(b)
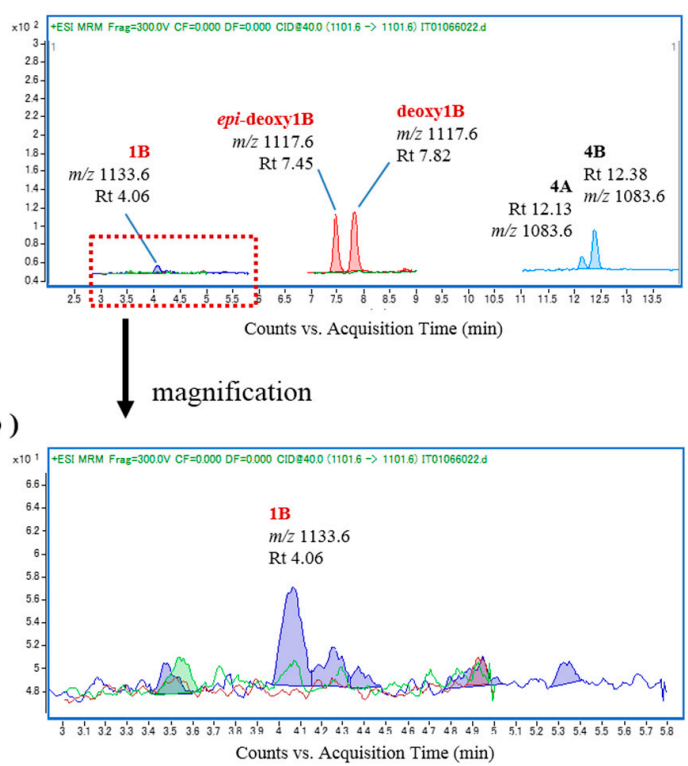

(c)

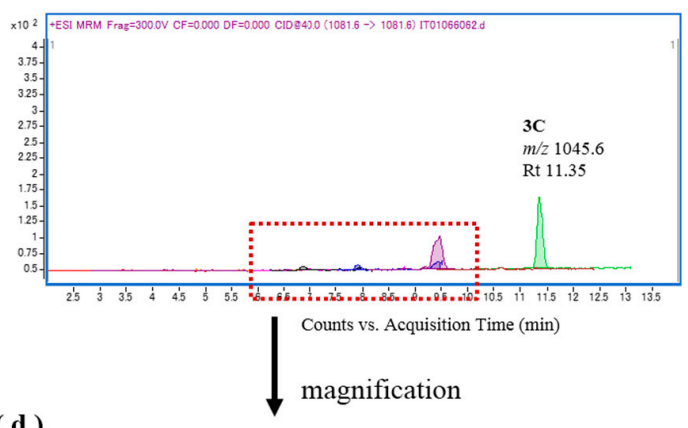

(d)

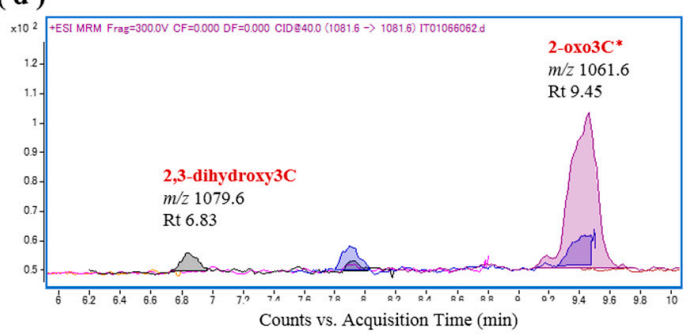

Figure 7. Chromatograms of the in vitro oxidation products of ciguatoxin-4A/4B (CTX4A/4B) and CTX3C following incubation with the liver S9 fraction of L. gibbus; $(\mathbf{a}, \mathbf{b})$ L. gibbus liver S9 and CTX4A/4B; and (c,d) L. gibbus liver S9 and CTX3C.
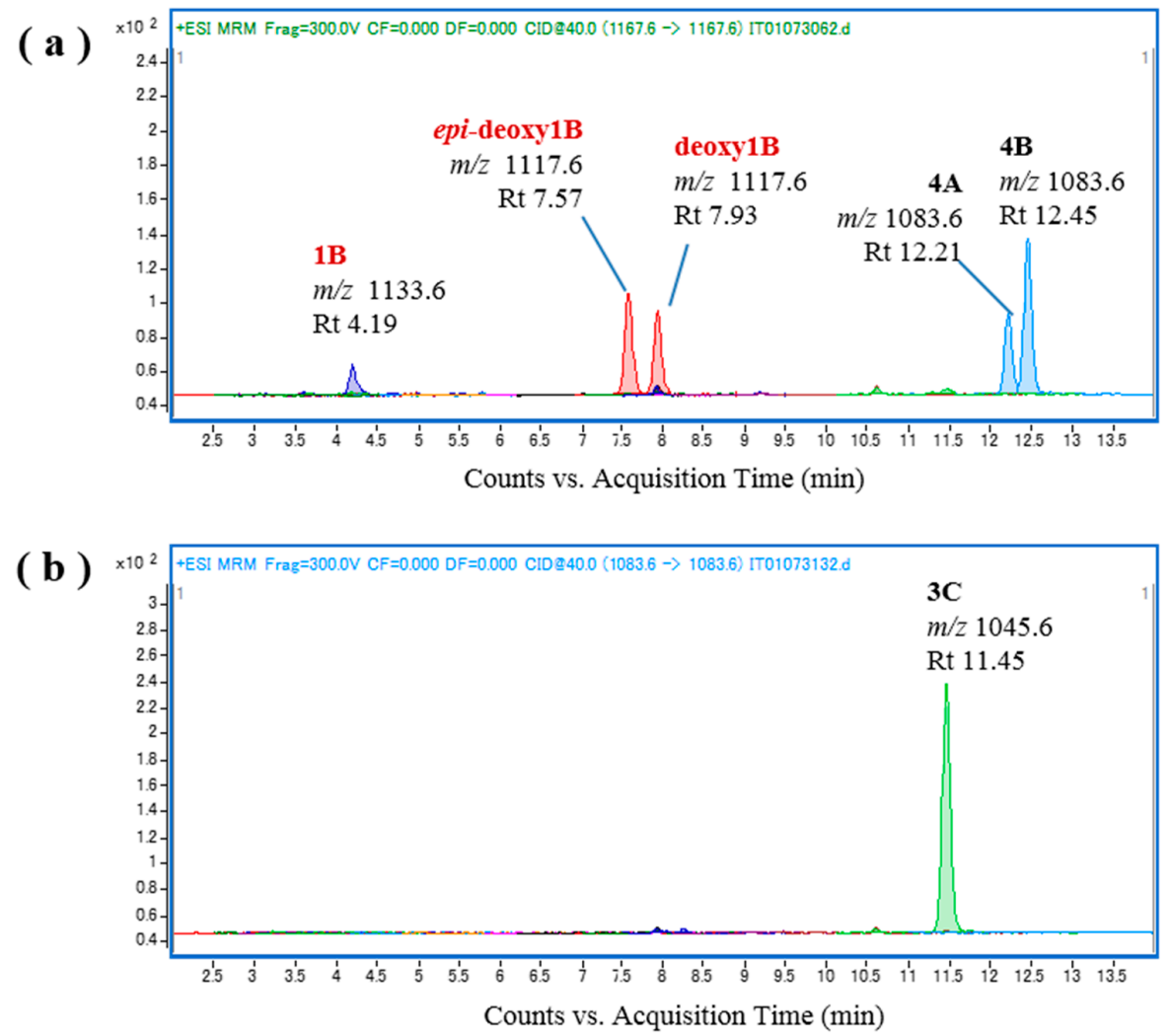

Figure 8. Cont. 


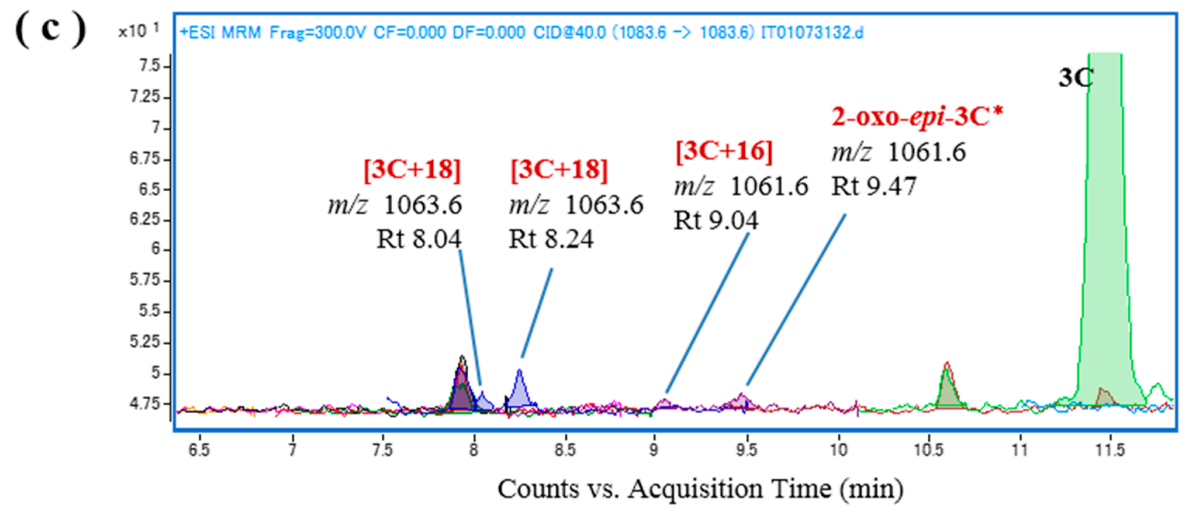

Figure 8. Chromatograms of the in vitro oxidation products of ciguatoxin-4A/4B (CTX4A/4B) and CTX3C following incubation with the liver S9 fraction of L. fulviflamma; (a) L. fulviflamma liver S9 and CTX4A/4B; and (b,c) L. fulviflamma liver S9 and CTX3C.

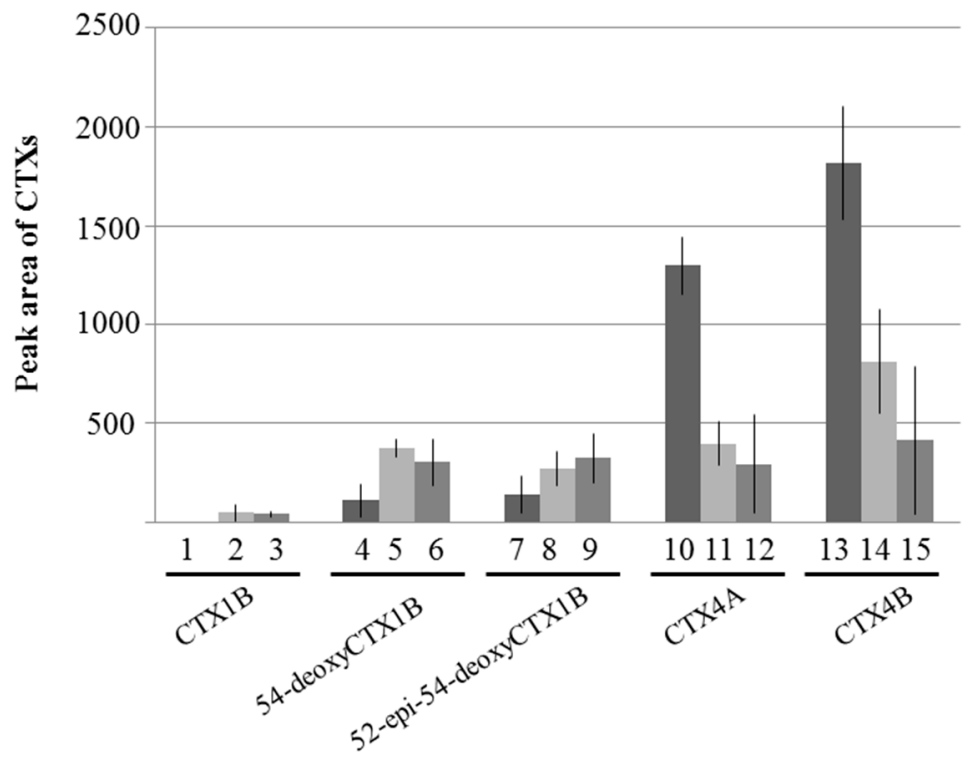

Figure 9. Comparison of the peak areas of various ciguatoxins (CTXs). CTX4A (5 ng) and CTX4B (7.5 ng) were incubated with fish liver S9 at $37^{\circ} \mathrm{C}$ for $60 \mathrm{~min}$ and the reaction products were identified by liquid chromatography-tandem mass spectrometry (LC-MS/MS) analysis using reference toxins. $1,4,7,10,13$ : the in vitro oxidation products following incubation with L. bohar liver S9. 2, 5, 8, 11, 14: the in vitro oxidation products following incubation with the liver S9 fraction of L. gibbus; and 3, 6, 9, 12,15 : the in vitro oxidation products following incubation with the liver S9 fraction of L. fulviflamma. Values represent the means $(n=3$ specimens/species $) \pm \mathrm{SD}$.

\section{Discussion}

The bio-oxidation of CTXs has received little attention to date due to the limited availability of purified toxins and the difficulty in developing an appropriate analytical method. In this study, we examined the mechanism that leads to characteristic toxin profiles in ciguateric fishes. We found that both fish liver S9 and human CYP3A4 converted CTX4A and CTX4B to CTX1B, 54-deoxyCTX1B, and 52-epi-54-deoxyCTX1B in vitro through oxidation reactions that occurred at the terminal parts of the molecules (Figure 10). This is the first study to provide insight into the biooxidation of CTXs using an enzymatic approach in vitro, providing a basis for further research into the metabolism of CTXs in fish and humans. 


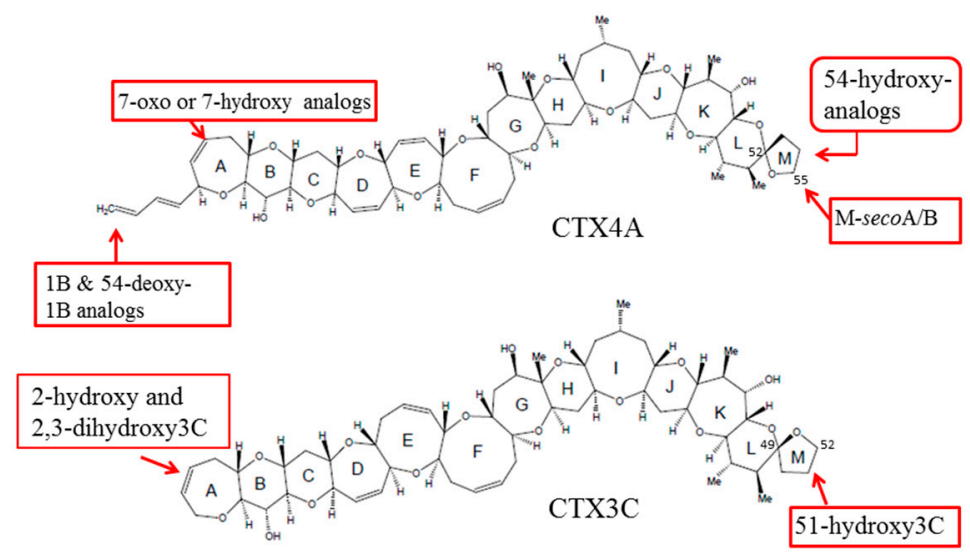

Figure 10. The position and mode of oxidation of ciguatoxin-4A (CTX4A) and CTX3C in recombinant human CYP3A4 (rhCYP3A4) and fish liver S9 fractions.

Uno et al. [21] previously provided an overview of the CYP superfamily in fish, but the relationship between these enzymes and CTX metabolism remained unclear. In the present study, we evaluated the feasibility of using fish liver S9 and rhCYP3A4 for in vitro oxidation of CTXs, and demonstrated that each of these as well as further fractionated microsomes successfully oxidized these toxins. These findings suggest that a CYP family enzyme in the fish liver is probably involved in the bio-oxidation of CTXs and that CTXs may also be bio-oxidized in the human liver following the consumption of contaminated fish.

CTX1B is the most potent CTX that is currently known with many bioactivities [16] and is the main congener that is found in carnivorous fish. It is believed that this fish metabolite is derived from the oxidation of CTX4A and CTX4B, which are produced by G. toxicus, and so the toxicity of CTXs to patients would be increased if this oxidation process occurs in the human liver. In this study, we detected M-seco-CTXs and 2-hydroxy analogs as the in vitro oxidation products, the signals for which were assigned based on previous data $[10,15]$. The toxicity of M-seco analogs is unknown but is presumed to be very low because they were isolated from the MBA negative fractions, and so they can be regarded as detoxification products.

In this study, we used four fish species from the genus Lutjanus (L. bohar, L. monostigma, L. gibbus, and L. fulviflamma). Among these, L. bohar is most frequently associated with CFP in Okinawa [21] and other regions [22] of the Pacific, while L. monostigma is reputed to be the most toxic fish species in Okinawa. By contrast, no episodes of CFP have been associated with L. gibbus or L. fulviflamma in Okinawa [21]. Consequently, L. bohar and L. monostigma are known as ciguateric fishes, while L. gibbus and L. fulviflamma are known as non-ciguateric fishes in Okinawa. A previous phylogenetic analysis using DNA barcode data of the cytochrome oxidase subunit I and 16S rRNA genes indicated that L. gibbus is more closely related to L. bohar than to L. fulviflamma [23]. However, comparison of the in vitro CTX oxidation activities in these fishes suggested that $L$. bohar and L. gibbus have different metabolic pathways for CTX detoxification, which is assumed to begin with an oxidation reaction; and these differences may lead to species-specific toxin profiles in fish. Furthermore, comparison of the liver S9 fractions prepared from five specimens of $L$. bohar demonstrated that there are also individual differences in CTX oxidation activity in this species-though it should be noted that these S9 fractions were prepared from fish obtained from commercial sources and so fluctuations in enzyme activity may have occurred.

Three major ciguatoxins (CTX3C, 51-hydroxyCTX3C, and CTX1B) have been successfully synthesized in previous studies [24-26]. However, the preparation of oxidative analogs, such as 54-deoxyCTX1B, 2-hydroxyCТХ3C, and 2,3-dihydroxyСТХЗ $\mathrm{C}$ has required their purification from ciguateric fish to date. By contrast, in the present study, CTX1B, 52-epi-54-deoxyCTX1B, 54-deoxyCTX1B, 2-hydroxyCTX3C, and 2,3-dihydroxyCTX3C were successfully produced in vitro by enzymatic oxidation, although further 
improvements may be required for their practical use. Thus, enzymatic preparation of reference toxins to be used in LC-MS analysis from the toxins produced by G. toxicus appears to be possible, but only after rigorous testing for optimum conditions and enzymes.

\section{Materials and Methods}

\subsection{Reagents and Materials}

CTX3C and a mixture of CTX4A and CTX4B were prepared from wild and cultured Gambierdiscus toxicus dinoflagellates [7,9,11,12]. A standard solution of a CTX mixture containing the toxins shown in Figure S1 was prepared using purified or semi-purified toxins from natural sources and identified by spectral analysis [7,9-13]. The methanol that was used for sample preparation and the LC-MS/MS mobile phase was of LC-MS grade (Kanto Chemical Co., Inc., Tokyo, Japan), while the ammonium formate solution $(1 \mathrm{~mol} / \mathrm{L})$ and formic acid were of high-performance liquid chromatography (HPLC) grade (Wako Chemical Industry, Ltd., Osaka, Japan). Ultra-pure water was supplied by Milli-Q ${ }^{\circledR}$ Integral Water Purification System for Chemical Analysis (Millipore, Bedford, MA, USA). Supersomes human CYP3A4 + reductase + b5 and the NADPH regenerating system were obtained from Corning (Corning Inc., Corning, NY, USA).

\subsection{Preparation of Fish Liver S9 and Microsomal Fractions}

Eighteen fish specimens from three representative ciguateric fish species (L. monostigma, L. bohar, and O. punctatus) and two non-ciguateric fish species (L. gibbus and L. fulviflamma) were collected from the Ryukyu Islands in Okinawa Prefecture. The liver was removed from each fish and promptly used for S9 fraction preparation. Individual liver samples were weighed and homogenized in two times the volume of ice-cold buffer $(20 \mathrm{mM}$ Tris, $0.15 \mathrm{M} \mathrm{KCl}, 2 \mathrm{mM}$ ethylenediaminetetraacetic acid (EDTA), $0.5 \mathrm{mM}$ benzamidine, $0.5 \mathrm{mM}$ dithiothreitol (DTT), $0.2 \mathrm{mM}$ phenylmethylsulfonyl fluoride (PMSF), and 10\% glycerol) using a glass-Teflon homogenizer. The homogenate was then centrifuged at $9000 \times g$ for $10 \mathrm{~min}$ at $4{ }^{\circ} \mathrm{C}$ and the resultant supernatant (S9 fraction) was ultracentrifuged at $100,000 \times g$ for $60 \mathrm{~min}$ at $4^{\circ} \mathrm{C}$. The microsome pellet was resuspended and homogenized in ice-cold buffer. The protein was assayed with a commercially available kit (TaKaRa Bradford Protein Assay Kit; TaKaRa, Japan) according to the manufacturer's instructions, using bovine serum albumin as a standard. The S9 and microsomal fractions were then stored in small aliquots at $-80^{\circ} \mathrm{C}$ until required for use. All preparation steps of the enzyme sources were carried out on ice.

\subsection{In Vitro CTX Oxidation Assay and Sample Preparation for LC-MS/MS Analysis}

For the CTX oxidation studies, the incubation mixture $(100 \mu \mathrm{L})$ included the following compounds: potassium phosphate buffer (0.1 M, pH 7.4); NADPH regeneration system; CTX4A (5 ng) + CTX4B (7.5 ng) or CTX3C (0.5 ng); and fish S9 (0.8 mg protein $/ \mathrm{mL})$, fish microsome (0.8 $\mathrm{mg}$ protein $/ \mathrm{mL})$, or recombinant human CYP3A4 $(0.1 \mathrm{mg}$ protein $/ \mathrm{mL})$. The reaction mixture was incubated at $37^{\circ} \mathrm{C}$ for $60 \mathrm{~min}$ and the reaction was then terminated by the addition of $100 \mu \mathrm{L}$ of acetonitrile. Following this, the mixture was centrifuged, the supernatant was dried, and the residues were re-solubilized in $1 \mathrm{~mL}$ of $50 \% \mathrm{MeOH}(v / v)$ and applied to an InertSep C18 (100 mg/1 mL) cartridge column (GL Science Inc., Tokyo, Japan) that had previously been treated with $3 \mathrm{~mL}$ of methanol and $3 \mathrm{~mL}$ of ultra-pure water. The column was washed with $3 \mathrm{~mL}$ of $60 \%$ methanol $(v / v)$ and the CTXs were eluted with $2 \mathrm{~mL}$ of methanol. The eluates were then dried under a nitrogen stream at $40{ }^{\circ} \mathrm{C}$ and dissolved in $1 \mathrm{~mL}$ of methanol in preparation for LC-MS/MS analysis.

\subsection{LC-MS/MS Analysis}

The LC-MS/MS system was comprised of an Agilent 1290 HPLC system and an Agilent 6460 Triple Quadrupole MS instrument (Agilent Technologies, Santa Clara, CA, USA). The LC-MS conditions matched those outlined previously $[15,16,27]$ with slight modification. 
Each sample solution $(5 \mu \mathrm{L})$ was injected into and separated with a Zorbax Eclipse Plus C18 column ( $2.1 \times 50 \mathrm{~mm}, 1.8 \mu \mathrm{m}$; Agilent Technologies, Santa Clara, CA, USA) at $40{ }^{\circ} \mathrm{C}$ using mobile phases $\mathrm{A}(5 \mathrm{mM}$ ammonium formate and $0.1 \%$ formic acid in water) and $\mathrm{B}(\mathrm{MeOH})$. The gradient started at $60 \% \mathrm{~B}$ and was held from 0.00 to $0.25 \mathrm{~min}$; it was then increased to $60-75 \% \mathrm{~B}$ from 0.25 to $0.50 \mathrm{~min}$ and $75-90 \% \mathrm{~B}$ from 0.5 to $12.0 \mathrm{~min}$, where it was held until $14.0 \mathrm{~min}$ with a flow rate of $0.4 \mathrm{~mL} / \mathrm{min}$. The analytical column was washed with methanol for $6 \mathrm{~min}$ and then re-equilibrated with $60 \%$ B for 3 min with a flow rate of $0.5 \mathrm{~mL} / \mathrm{min}$.

The MS parameters were set as follows: electrospray ionization (ESI) positive mode with Agilent Jet Stream; dry gas, $10 \mathrm{~L} / \mathrm{min}$ of $\mathrm{N}_{2}$ at $300{ }^{\circ} \mathrm{C}$; nebulizer gas, $\mathrm{N}_{2}$ at $50 \mathrm{psi}$; sheath gas, $11 \mathrm{~L} / \mathrm{min}$ of $\mathrm{N}_{2}$ at $400{ }^{\circ} \mathrm{C}$; capillary voltage, $5000 \mathrm{~V}$; nozzle voltage, $1000 \mathrm{~V}$; and fragmentor voltage, $300 \mathrm{~V}$. The $[\mathrm{M}+\mathrm{Na}]^{+}$ ions were used as precursor ions and product ions, with a collision energy of $40 \mathrm{eV}$, as previously reported $[15,16,27]$.

Supplementary Materials: The following are available online at www.mdpi.com/2072-6651/9/7/205/s1. Figure S1: Chromatograms, retention times (Rts), and $m / z$ values for the ciguatoxins (CTXs) that were used as substrates for in vitro oxidation; Figure S2: Chromatograms of the in vitro oxidation products of ciguatoxin-4A/4B (CTX4A/4B) following incubation with recombinant human CYP3A4 (rhCYP3A4); Figure S3: Chromatograms of the in vitro oxidation products of ciguatoxin- $4 \mathrm{~A} / 4 \mathrm{~B}(\mathrm{CTX} 4 \mathrm{~A} / 4 \mathrm{~B})$ following incubation with human liver microsomes; Figure S4: Chromatograms of the in vitro oxidation products of ciguatoxin-4A/4B (CTX4A/4B) following incubation with the S9 fractions from four different Lutjanus bohar specimens; Figure S5: Chromatograms of the S9 fractions of four different Lutjanus bohar specimens; Table S1: Body size of fish samples used in this study.

Acknowledgments: This work was supported by Grants from Health Labour Sciences Research and JSPS KAKENHI (15K07594).

Author Contributions: T.I. and T.Y. conceived and designed the experiments; T.I., K.K. and N.O. performed the experiments; T.I., K.K. and N.O. analyzed the data; T.I., K.K., N.O. and T.Y. contributed reagents/materials/analysis tools; and T.I. and T.Y. wrote the paper.

Conflicts of Interest: The authors declare no conflict of interest.

\section{References}

1. Yasumoto, T. The chemistry and biological function of natural marine toxins. Chem. Rec. 2001, 1, 228-242. [CrossRef] [PubMed]

2. Isbister, G.K.; Kiernan, M.C. Neurotoxic marine poisoning. Lancet Neurol. 2005, 4, 219-228. [CrossRef]

3. Friedman, M.A.; Fleming, L.E.; Fernandez, M.; Bienfang, P.; Schrank, K.; Dickey, R.; Bottein, M.Y.; Backer, L.; Ayyar, R.; Weisman, R.; et al. Ciguatera fish poisoning: Treatment, prevention and management. Mar. Drugs 2008, 6, 456-479. [CrossRef] [PubMed]

4. Skinner, M.P.; Brewer, T.D.; Johnstone, R.; Fleming, L.E.; Lewis, R.J. Ciguatera fish poisoning in the pacific islands (1998 to 2008). PLoS Negl. Trop. Dis. 2011, 5, e1416. [CrossRef] [PubMed]

5. Grattan, L.M.; Holobaugh, S.; Morris, J.G., Jr. Harmful algal blooms and public health. Harmful Algae 2016, 57, 2-8. [CrossRef] [PubMed]

6. Yasumoto, T.; Murata, M. Marine toxins. Chem. Rev. 1993, 93, 1897-1909. [CrossRef]

7. Murata, M.; Legrand, A.M.; Ishibashi, Y.; Fukui, M.; Yasumoto, T. Structures and configurations of ciguatoxin from the moray eel Gymnothorax javanicus and its likely precursor from the dinoflagellate Gambierdiscus toxicus. J. Am. Chem. Soc. 1990, 112, 4380-4386. [CrossRef]

8. Chinain, M.; Darius, H.T.; Ung, A.; Cruchet, P.; Wang, Z.; Ponton, D.; Laurent, D.; Pauillac, S. Growth and toxin production in the ciguatera-causing dinoflagellate Gambierdiscus polynesiensis (Dinophyceae) in culture. Toxicon Off. J. Int. Soc. Toxinol. 2010, 56, 739-750. [CrossRef] [PubMed]

9. Murata, M.; Legrand, A.M.; Ishibashi, Y.; Yasumoto, T. Structures of ciguatoxin and its congener. J. Am. Chem. Soc. 1989, 111, 8929-8931. [CrossRef]

10. Yasumoto, T.; Igarashi, T.; Legrand, A.-M.; Cruchet, P.; Chinain, M.; Fujita, T.; Naoki, H. Structural elucidation of ciguatoxin congeners by fast-atom bombardment tandem mass spectroscopy. J. Am. Chem. Soc. 2000, 122, 4988-4989. [CrossRef]

11. Satake, M.; Murata, M.; Yasumoto, T. The structure of CTX3C, a ciguatoxin congener isolated from cultured Gambierdiscus toxicus. Tetrahedron Lett. 1993, 34, 1975-1978. [CrossRef] 
12. Satake, M.; Ishibashi, Y.; Legrand, A.M.; Yasumoto, T. Isolation and structure of ciguatoxin-4a, a new ciguatoxin precursor, from cultures of dinoflagellate Gambierdiscus toxicus and parrotfish Scarus gibbus. Biosci. Biotechnol. Biochem. 1996, 60, 2103-2105. [CrossRef] [PubMed]

13. Satake, M.; Fukui, M.; Legrand, A.-M.; Cruchet, P.; Yasumoto, T. Isolation and structures of new ciguatoxin analogs, 2,3-dihydroxyCTX3C and 51-hydroxyCTX3C, accumulated in tropical reef fish. Tetrahedron Lett. 1998, 39, 1197-1198. [CrossRef]

14. Lewis, R.J. Ciguatera: Australian perspectives on a global problem. Toxicon Off. J. Int. Soc. Toxinol. 2006, 48, 799-809. [CrossRef] [PubMed]

15. Yogi, K.; Oshiro, N.; Inafuku, Y.; Hirama, M.; Yasumoto, T. Detailed LC-MS/MS analysis of ciguatoxins revealing distinct regional and species characteristics in fish and causative alga from the Pacific. Anal. Chem. 2011, 83, 8886-8891. [CrossRef] [PubMed]

16. Yogi, K.; Sakugawa, S.; Oshiro, N.; Ikehara, T.; Sugiyama, K.; Yasumoto, T. Determination of toxins involved in ciguatera fish poisoning in the pacific by LC/MS. J. AOAC Int. 2014, 97, 398-402. [CrossRef] [PubMed]

17. Anzenbacher, P.; Anzenbacherova, E. Cytochromes p450 and metabolism of xenobiotics. Cell. Mol. Life Sci. CMLS 2001, 58, 737-747. [CrossRef] [PubMed]

18. Bjornsson, T.D.; Callaghan, J.T.; Einolf, H.J.; Fischer, V.; Gan, L.; Grimm, S.; Kao, J.; King, S.P.; Miwa, G.; $\mathrm{Ni}$, L.; et al. The conduct of in vitro and in vivo drug-drug interaction studies: A PhRMA perspective. J. Clin. Pharm. 2003, 43, 443-469. [CrossRef]

19. Fisher, M.B.; Campanale, K.; Ackermann, B.L.; VandenBranden, M.; Wrighton, S.A. In vitro glucuronidation using human liver microsomes and the pore-forming peptide alamethicin. Drug Metab. Dispos. Boil. Fate Chem. 2000, 28, 560-566.

20. Spatzenegger, M.; Jaeger, W. Clinical importance of hepatic cytochrome p450 in drug metabolism. Drug Metab. Rev. 1995, 27, 397-417. [CrossRef] [PubMed]

21. Oshiro, N.; Yogi, K.; Asato, S.; Sasaki, T.; Tamanaha, K.; Hirama, M.; Yasumoto, T.; Inafuku, Y. Ciguatera incidence and fish toxicity in Okinawa, Japan. Toxicon Off. J. Int. Soc. Toxinol. 2010, 56, 656-661. [CrossRef] [PubMed]

22. Wong, C.K.; Hung, P.; Lo, J.Y. Ciguatera fish poisoning in Hong Kong-A 10-year perspective on the class of ciguatoxins. Toxicon Off. J. Int. Soc. Toxinol. 2014, 86, 96-106. [CrossRef] [PubMed]

23. Rajeish, M.; Malathi, S.; Madhushree, H.N.; Venugopal, M.N. Presumptive case of ciguatera fish poisoning in Mangalore, India. Curr. Sci. 2016, 111, 1543-1547. [CrossRef]

24. Hirama, M.; Oishi, T.; Uehara, H.; Inoue, M.; Maruyama, M.; Oguri, H.; Satake, M. Total synthesis of ciguatoxin CTX3C. Science 2001, 294, 1904-1907. [CrossRef] [PubMed]

25. Inoue, M.; Miyazaki, K.; Uehara, H.; Maruyama, M.; Hirama, M. First- and second-generation total synthesis of ciguatoxin CTX3C. Proc. Natl. Acad. Sci. USA 2004, 101, 12013-12018. [CrossRef] [PubMed]

26. Inoue, M.; Miyazaki, K.; Ishihara, Y.; Tatami, A.; Ohnuma, Y.; Kawada, Y.; Komano, K.; Yamashita, S.; Lee, N.; Hirama, M. Total synthesis of ciguatoxin and 51-hydroxyCTХ3C. J. Am. Chem. Soc. 2006, 128, 9352-9354. [CrossRef] [PubMed]

27. Yogi, K.; Oshiro, N.; Matsuda, S.; Sakugawa, S.; Matsuo, T.; Yasumoto, T. Toxin profiles in fish implicated in ciguatera fish poisoning in Amami and Kakeroma islands, Kagoshima prefecture, Japan. Food Hyg. Saf. Sci. (Shokuhin Eiseigaku Zasshi) 2013, 54, 385-391. [CrossRef]

(C) 2017 by the authors. Licensee MDPI, Basel, Switzerland. This article is an open access article distributed under the terms and conditions of the Creative Commons Attribution (CC BY) license (http://creativecommons.org/licenses/by/4.0/). 